\title{
Self-induced electrostatic-boosted radioisotope heat sources
}

\author{
Francisco J. Arias*, Geoffrey T. Parks \\ Department of Engineering, University of Cambridge, Trumpington Street, Cambridge, CB2 1PZ, United Kingdom
}

\section{A R T I C L E I N F O}

\section{Article history:}

Received 5 March 2015

Received in revised form 25 May 2015

Accepted 12 June 2015

Available online 4 July 2015

\section{Keywords:}

Nuclear batteries

Radioisotope heater units

Electrostatic potential

\begin{abstract}
A B S T R A C T
In this paper the possibility of stimulated self-induced electrostatic fields in radioisotope heat sources for power enhancement is discussed. Because electrons have higher mobility than the positively charged fragments from radioactive decay, a build-up of positive charges can be promoted, leading to an internal induced electrostatic field. This, in turn, results in a repulsive force acting on positive charges, endowing them with additional kinetic energy, and heat release after these charged particles are stopped by inelastic collisions with the boundary wall. Utilizing a simplified geometrical model, an analytical expression for the attainable power enhancement is derived. It is shown that the proposed concept could result in power enhancements of $5-10 \%$ for beta sources but enhancements are negligible for alpha sources.
\end{abstract}

() 2015 Elsevier Ltd. All rights reserved.

\section{Introduction}

Radioisotope Heat Sources (RHSs) are small devices that provide heat through radioactive decay. The heat produced by these devices is released continuously for several decades and, theoretically, depending on the half-lives of the isotopes used, for up to a century or more. The heat produced from RHSs can be used directly to keep structures, systems, and instruments warm enough to operate effectively; such devices are called Radioisotope Heater Units (RHUs). Alternatively, the heat released by RHSs can be converted into electricity by the Seebeck effect; in this case, such devices are called Radioisotope Thermoelectric Generators (RTGs).

The objective of this work was to analyse a new approach based on a self-induced electrostatic field. However, the electrostatic field is not desired for extracting direct electrical energy (as in the case of nuclear batteries), but instead to transform the gained electrostatic potential of the charged particles into heat by increasing their kinetic energy and thus making more vigorous their collisions with their surroundings. In what follows, we will outline the essential ideas behind this concept, and we will derive an analytical expression for the attainable energetic gain using this mechanism.

Although, in rigorous terms, all radioisotopic generators are inevitably heat sources because a fraction of the energy from the radiative decay will always become heat, in this paper we define RHSs to be devices the ultimate goal of which is to maximize the

\footnotetext{
* Corresponding author.

E-mail address: fja30@cam.ac.uk (F.J. Arias).
}

fraction of energy released as heat. This definition establishes a clear distinction from conventional nuclear batteries based on nonthermal converters, the ultimate goal of which is to harness the motion of charged particles to produce an electrical potential.

Fig. 1 shows the range of radioisotope-based generators used for energy production. In this figure, we can see two clear divisions in the use of radioisotope generators. On one hand, there is their use as nuclear batteries, the ultimate goal of which is the production of electricity. These can be sub-classified based on the way in which the electrical conversion is performed: namely as non-thermal converters (taking advantage of the direct motion of charged particles), or thermal converters as in the case of RTGs. On the other hand, radioisotope generators can be used as heater units, the ultimate goal of which is the production of heat. There are many applications for RHSs in terrestrial and airborne systems and, more significantly, in space applications, because of their intrinsic high reliability not only in the production of heat but also in the design of energy extraction systems. Suffice it to say that the simplicity of RHSs allows easy heat extraction by using, for example, an optimal spacing between parallel and flat plates (Bejan and Sciubba, 1992; Hajmohammadi et al., 2013a) or tubular geometries (Hajmohammadi et al., 2013b) and temperature control can be performed by simple wall-thickness and material control (Hajmohammadi et al., 2012, 2013c, 2015).

In this paper we are interested only in RHSs, which include RHUs as well as RTGs. However, we are only concerned with enhancement of the heat released from radiative decay, so for the particular case of RTGs we do not consider the process of thermal 


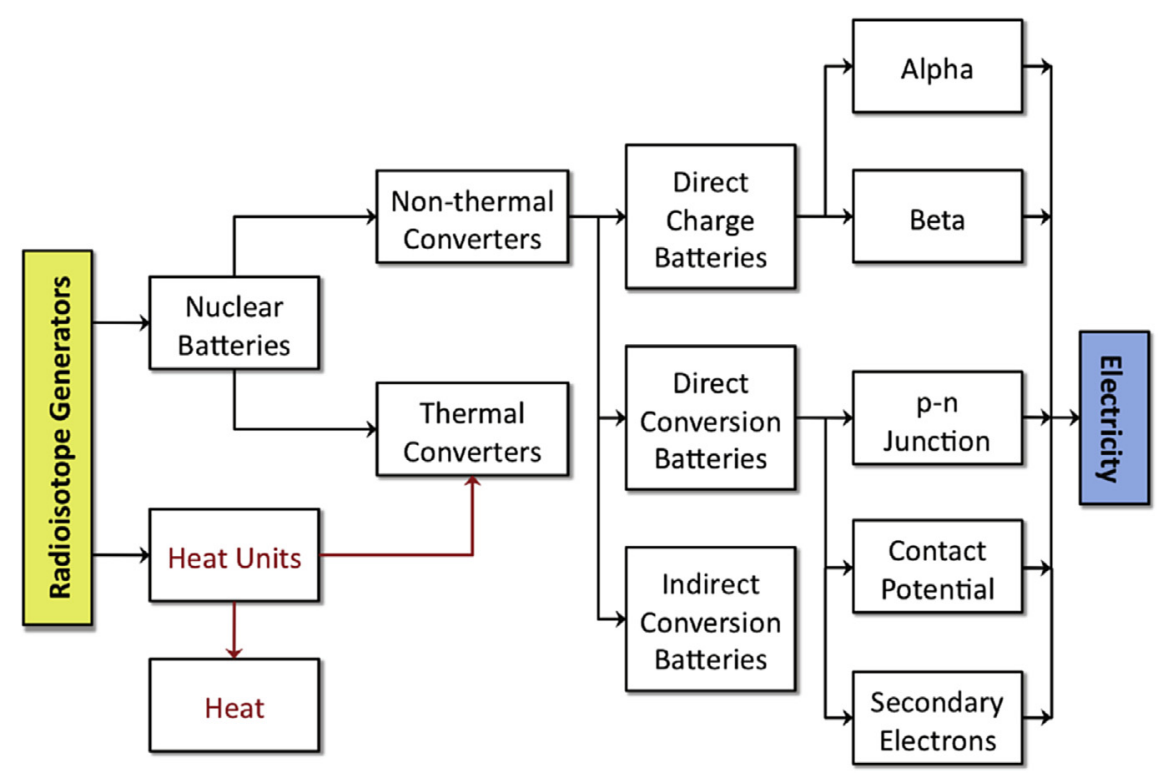

Fig. 1. The energetic uses of radioisotope generators.

conversion into electricity, which will be essentially the same as for classical RTGs but now with an enhanced heat source, i.e. at a higher temperature, giving better Seebeck effect performance.

The performance of non-thermal-type nuclear batteries is governed by the length scales of the system, namely the range of ionizing radiation and the size of the transducer, and can therefore be improved by geometrical design (Prelas et al., 2014). In contrast, the performance of thermal-type nuclear generators, such as RHUs, basically depends on the kind of radioisotope(s) used. Once the choice of the kind of radioisotope to be used in the RHU has been made, the heat per unit mass is fixed. This feature of RHUs, while giving them their recognised reliability, also limits the scope for improving their performance or that of the heat source in RTGs. As a result, research on RHUs was for many years limited to the selection of the best radioisotope. Most recently, there have been attempts to improve the efficiency of RHU and RTG sources by the use of hybrid designs stimulating parallel fission reactions in subcritical radioisotope heat sources (Wang and He, 2014; Arias, 2011). For the interested reader, an up-to-date, extensive review of the state of the art for nuclear batteries can be found in Prelas et al. (2014) and Radioisotope Power Systems Committee and National Research Council (2009), and the basic principles and parameters of radionuclide generators are well described in Lazarenko et al. (1988), Anderson et al. (2005) and Corliss and Harvey (1964).

The physical concept proposed in the present work is conceptually very intuitive. An electrostatic-boosted radioisotope heat source can be summarised as a classical radioisotope heat source, which provides heat through radioactive decay, but which is further enhanced by a self-stimulated electrostatic field. The reader can visualise and acquire a quick understanding of the proposed concept through its similarity to the well-known phenomenon of fuel fleas, because they are, in essence, based on exactly the same principle, i.e. the asymmetric distribution of charges leading to the creation of an electrostatic field and a repulsive force.

Fig. 2 depicts the phenomenon known as fuel fleas. In summary, fuel fleas are microscopic hot particles of new or spent nuclear fuel. Although small, they tend to be intensely radioactive. The fuel particles, the size of which is about $10 \mu \mathrm{m}$, are a strong source of beta and gamma radiation and a weaker source of alpha radiation.

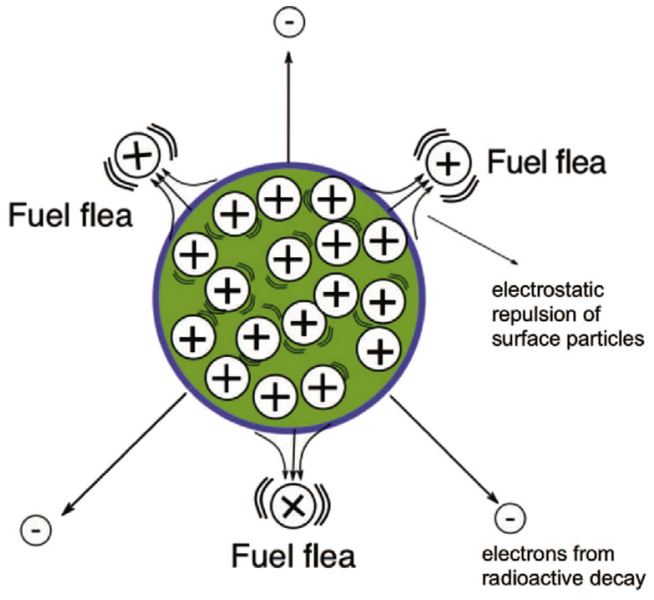

Fig. 2. The fuel flea phenomenon: fuel particles lose more negatively charged particles than positively charged ones leading to a build-up of positive electrostatic charge on the particle, causing it to "jump" from surface to surface and easily become airborne.

The disparity between alpha and beta radiation levels (alpha activity is typically 100-1000 times weaker than beta, so the fuel particle loses many more negatively charged particles than positively charged ones) leads to a build-up of positive electrostatic charge on the particle, causing the particle to "jump" from surface to surface and easily become airborne. Another mechanism associated with the build-up of positive electrostatic charge due to the higher mobility of negative charges is the so-called aggregate-recoil phenomenon (Trenn, 1980). This phenomenon has been suggested as the possible cause of the anomalous volatility and ability easily to become airborne exhibited by some alpha sources, such as polonium-210. If polonium-210 is heated in air to $55^{\circ} \mathrm{C}, 50 \%$ of the sample vaporizes in $45 \mathrm{~h}$ to form diatomic $\mathrm{Po}_{2}$ molecules, even though the melting point of polonium is $254^{\circ} \mathrm{C}$ and its boiling point is $962^{\circ} \mathrm{C}$. One suggestion of how polonium does this is that small clusters of polonium atoms are spalled off by the build-up of positive electrostatic charges.

Now, we will explain how the usually undesired phenomenon of fuel fleas can be harnessed to boost a nuclear heat source. 


\section{The electrostatic-boosted encapsulated radioisotope heat source}

Let us consider an encapsulated radioisotope heat source (ERHS) with a distribution and geometry as depicted in Fig. 3.

The ERHS is formed of three regions. Region I: The kernel with a radius $r=a$ where an aerosolized radioisotope source is placed and separated from the outer region. Region II: The shroud region of internal radius $r=a$ and external radius $r=b$, which contains the same aerosolized radioisotope source. Region III: The matrix: a region without any radioisotope source which acts as shielding and a centre for electronic recombination.

There are two reasons for the use of an aerosolized radioisotope:

1. It allows the most energetic electrons to escape from Region I and Region II and be absorbed in Region III.

2. In Region II it allows the free-mobilization of positive charges, then the direct conversion of electric potential energy into kinetic energy and then into heat released after collision at the interface, i.e. at $r=b$.

In summary, the essential idea behind the electrostatic-boosted radioisotope heat concept is this: In the internal core (Region I) there will be a build-up of positive charge because of the higher mobility of electrons in comparison with the positively charged particles. It is important to note that, although there will be substantial production of electron-ion pairs (from secondary knock-on reactions), conservatively we can assume that all these ions and electrons recombine, i.e. are neutralized, and only the most energetic electrons (which have higher path lengths) can escape from Region I and Region II to be absorbed in the matrix (Region III).

As a result of this build-up of positive charge, in Region I the positive ions are symmetrically repelled by each other and pushed to the Region I-Region II interface, i.e. at $r=a$, where they will accumulate. Because of the high mobility of ions inside a gas, the core region is like a conductor, and it is well-known that in a spherical conductor charges are only located at the outer surface. Nevertheless, from an electrostatic point of view it does not matter if the positive charges are located at the surface $r=a$ or if they are volumetrically and homogeneously distributed inside the sphere of radius $r=a$, because the electrical potential generated in Region II will be the same as for a point charge concentrated at the origin $r=0$.

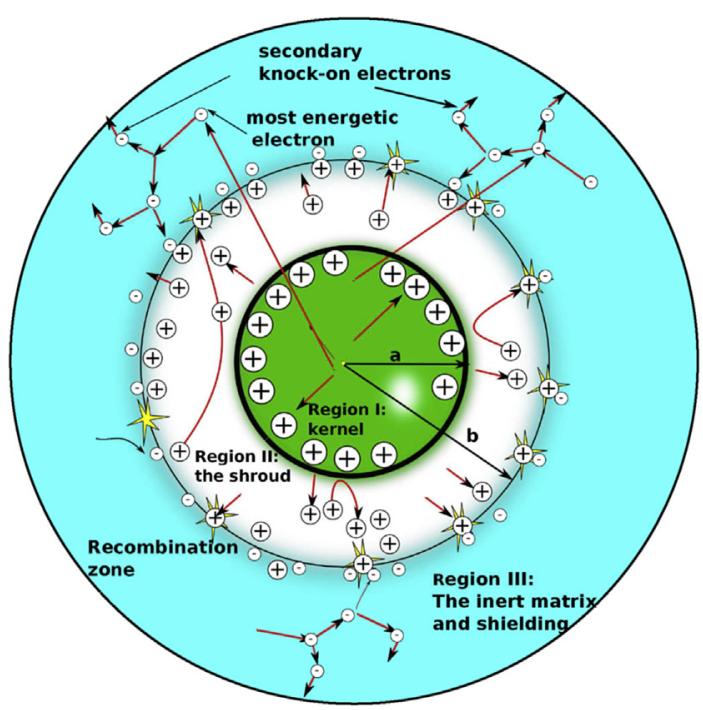

Fig. 3. Essentials of the electrostatic-boosted radioisotope heat source concept.
Now, after the accumulation of positive charges in Region I, an effective electrostatic field is developed acting radially on Region II. Thus, any positive charge created in Region II will be instantaneously pushed outwards by the repulsive electric field generated in Region I. This electrostatic potential will result in an increase in kinetic energy, which means that the electrostatically accelerated positive ions will deposit more energy as heat after inelastic collisions at the interface at $r=b$.

It is important to recall that, although we are exploiting the generation of a self-induced electric field, this field has nothing to do with its use in a nuclear electric battery. Here, in a very different way, the electrostatic field is created in order to promote more vigorous collisions of the charged ions, and this approach is, as far as the authors know, radically different from previous concepts.

Finally, the positive charges in Region II, which have been pushed towards the outer layer at $r=b$ (see Fig. 3), will impact and transfer their kinetic energy into heat. Secondary electrons coming from Region III will recombine and there will be no build-up of positive charge at the interface at $r=b$. However, recombination at the interface at $r=b$ with secondary electrons coming from Region III, although desirable, is not necessary. In fact, from Gauss' Law we know that the resultant electric field due to contributions of outside charges in producing flux is zero, meaning that, even if positive charge did accumulate at the boundary $r=b$, this would not affect the electrical field generated by the positive charges accumulated at the edge of Region I.

In the next section we derive an analytical expression for the attainable enhancement for an electrostatic-boosted radioisotope heat source.

\section{Statement of the concept}

Let us denote the total positive charge in Region I (depicted previously in Fig. 3) as $Q_{t}$. Then the electrical field in Region II generated by the internal charge in Region I is given by (Plonus, 1978):

$\mathbf{E}=\frac{Q_{t}}{4 \pi \varepsilon_{0} r^{2}} \quad r \geq a$

and the electrical potential is:

$\Phi=\int_{r}^{b} \mathbf{E} d r=\frac{Q_{t}}{4 \pi \varepsilon_{0}}\left[\frac{1}{r}-\frac{1}{b}\right] \quad r \geq a$

where $\varepsilon_{0}$ is the electrical permittivity in vacuum.

The positive charge $Q_{t}$ in the kernel (Region I) will attain its maximum value when it is producing a potential equal to the most energetic electron. At this point any electron (even the most energetic) will be unable to escape Region I and electronic recombination will neutralize any new production of positive charge. Therefore, the maximum total positive charge $Q_{t}$ is given by Eq. (1) integrated from $r=a$ to $r=\infty$ or

$Q_{t}=4 \pi \varepsilon_{0} a e_{m}$

where $e_{m}$ is the energy (in electron-volts) of the most energetic electron generated.

The electrostatic energy gained (which will be transformed into kinetic energy and then into heat by inelastic collisions) per unit electrical charge in Region II is given by Eq. (2). Therefore, the total energy $W_{e}$ is obtained by accounting for the total charge being produced, i.e. 
$W_{e}=\int_{a}^{b} \Phi \rho_{+} 4 \pi r^{2} d r$

where $\rho_{+}$is the volumetric density of charge in Region II.

Taking into account Eqs. (2) and (3) and integrating, Eq. (4) becomes:

$W_{e}=4 \pi a e_{m} \rho_{+}\left[\frac{b^{2}}{6}+\frac{a^{3}}{3 b}-\frac{a^{2}}{2}\right]$

Writing the external radius of Region II as a function of the inner radius as $b=x a$, then Eq. (5) becomes:

$W_{e}=\frac{4 \pi a^{3}}{3} e_{m} \rho_{+}\left[\frac{x^{2}}{2}+\frac{1}{x}-\frac{3}{2}\right]$

and the electrostatic power will be

$\dot{W}_{e}=\frac{4 \pi a^{3}}{3} e_{m} \dot{\rho}_{+}\left[\frac{x^{2}}{2}+\frac{1}{x}-\frac{3}{2}\right]$

where $\dot{\rho}_{+}$is the rate of creation of positive charge (per second).

The decay heat power from a traditional (not electrostatically enhanced) radioisotope heat source of the same geometry is given by:

$\dot{W}_{p}=\frac{4 \pi\left(b^{3}-a^{3}\right)}{3} N_{0} \lambda \exp ^{-\lambda t} e_{d} q$

where $N_{0}$ is the number of atoms of radioisotope per unit volume, $\lambda$ is the decay constant (in $\mathrm{s}^{-1}$ ), $t$ is time, $e_{d}$ is the associated energy from radioactive decay (in electron-volts), and $q$ is the charge of an electron.

The rate of creation of positive charge per unit volume, $\dot{\rho}_{+}$in Eq. (7), can be expressed as:

$\dot{\rho}_{+}=N_{0} \lambda \exp ^{-\lambda t} z q$

where $z$ is the number of electrical charges created per disintegration.

The total power $\dot{W}_{t}$ will be the sum of the direct power given by Eq. (8) and the electrostatic power given by Eq. (7):

$\dot{W}_{t}=\dot{W}_{p}+\dot{W}_{e}$

or

$\dot{W}_{t}=\dot{W}_{p}\left[1+z \frac{e_{m}}{e_{d}} \Delta\right]$

where

$\Delta=\left[\frac{x^{2}}{2}+\frac{1}{x}-\frac{3}{2}\right]\left[\frac{1}{x^{3}-1}\right]$

Then an electrostatic gain $\mathbf{G}_{e}$ may be defined as:

$\mathbf{G}_{e}=z \frac{e_{m}}{e_{d}} \Delta$

and Eq. (11) becomes:

$\dot{W}_{t}=\dot{W}_{p}\left[1+\mathbf{G}_{e}\right]$

It is interesting to see that, from an energetic point of view, we can interpret the result of Eq. (14) by saying that we are "replacing" our initial radioisotope by another with an effective energy per unit

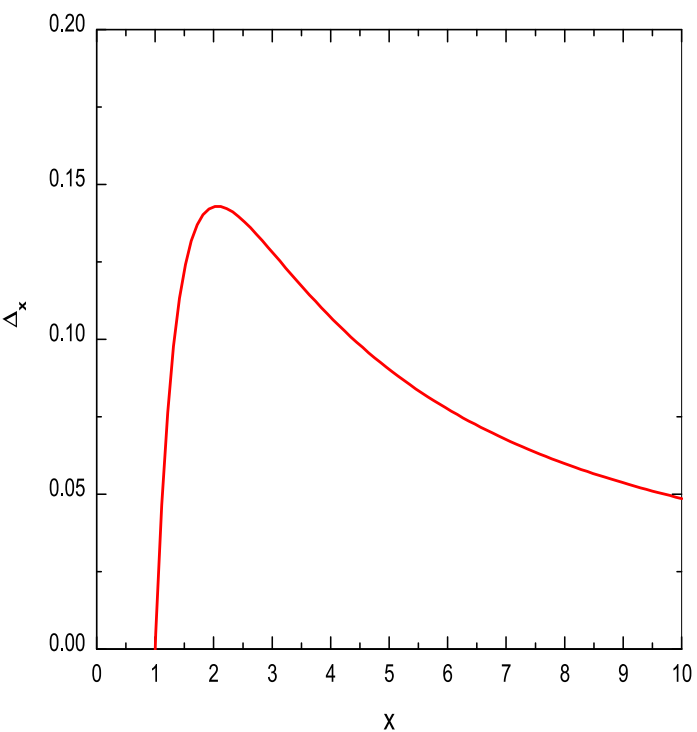

Fig. 4. The parameter $\Delta$ in Eq. (12) as a function of the ratio of the outer to inner radius $x=\frac{b}{a}$.

of volume enhanced by a factor of $\left[1+\mathbf{G}_{e}\right]$. So, from this point of view, the proposed concept can be used in current RHUs or RTGs without any overall change in the design of their converters.

\section{Evaluation of the proposed approach}

Fig. 4 shows the parameter $\Delta$ as a function of $x$. It is readily apparent that a maximum value is obtained when $x \approx 2$. This result means that, because the total diameter of the capsule must be at least equal to the mean path length of the most energetic electron $l_{e}$, then with $b \leq \frac{l_{e}}{2}$ the inner core which maximizes the electrostatic enhancement will have a radius $a=\frac{b}{2}=\frac{l_{e}}{4}$. Using this, a first estimation can be made on the size of the ERHS.

Many semi-empirical formulations are available for the range of electrons (or $\beta$ particles), but, in view of the uncertainty, the simplest expression, due to Katz and Penfold (Katz and Penfold 1952), seems preferable:

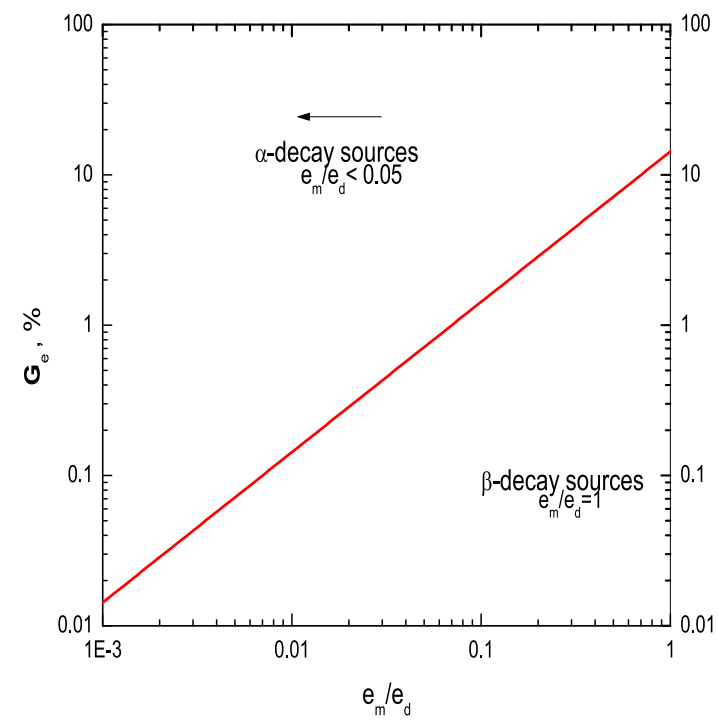

Fig. 5. The electrostatic gain $\mathbf{G}_{e}$ as a function of the ratio of the energy of the most energetic electron and the energy from the radioactive decay $\frac{e_{m}}{e_{d}}$. 

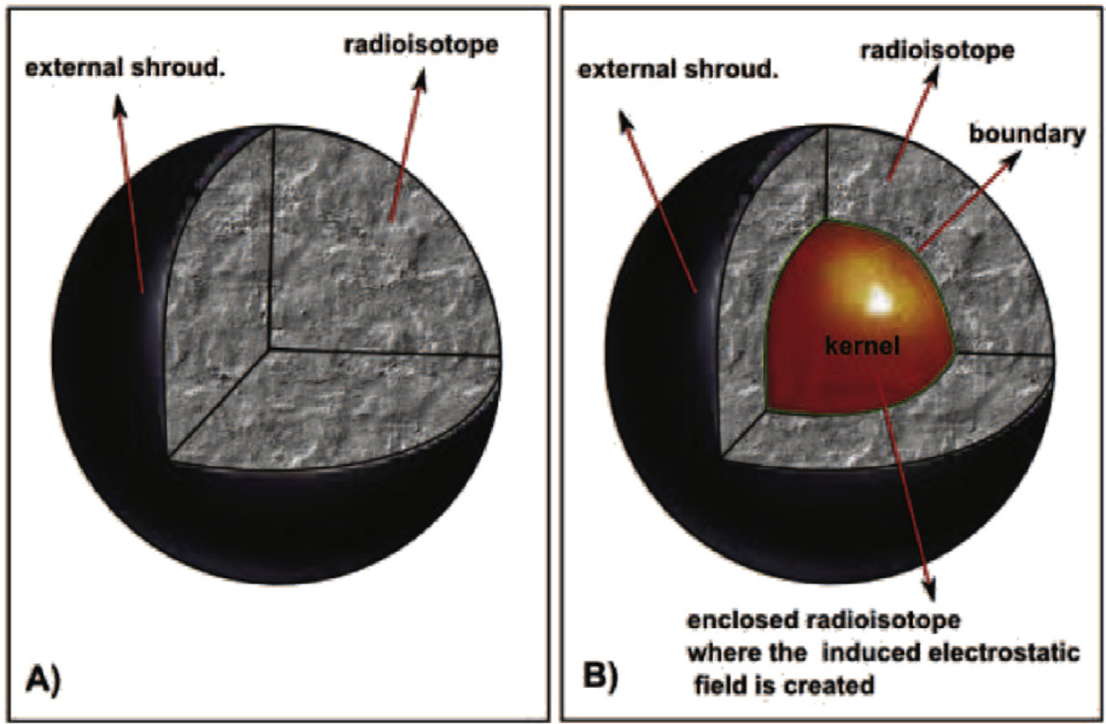

Fig. 6. A) A classical spherically encapsulated RHU; B) The same RHU enhanced electrostatically by separating an internal region or kernel from the outer region.

$l_{e}=0.412 \frac{e^{n}}{\rho} \quad 0.01<E<3.0 \mathrm{MeV}$

where $l_{e}$ is in $\mathrm{cm}, e$ is the energy of the electron in $\mathrm{MeV}, \rho$ is the density of the medium in $\mathrm{g} / \mathrm{cm}^{3}$, and

$n=1.265-0.0954 \ln e$

Empirical Eq. (16) is the most widely accepted relation and applies to the practical, or extrapolated, range of mono-energetic electrons. Thus, for $\beta$ particles of $\sim \mathrm{MeV}$ energies, as for example those emitted by caesium-137, and using a gas density of $0.1 \mathrm{~g} / \mathrm{cm}^{3}$, then $l_{e} \approx 5 \mathrm{~cm}$. So, for this case, the size of Region II of the ERHS will be $b \approx 2.5 \mathrm{~cm}$ and $a \approx 1.25 \mathrm{~cm}$.

Finally, using the value of $x$ which maximizes the electrostatic gain, i.e. $x \approx 2$, we obtain Fig. 5, which shows $\mathbf{G}_{e}$ as a function of the ratio $\frac{e_{m}}{e_{d}}$, where the most conservative value of parameter $z(z=1)$ was assumed.

Referring to Fig. 5 , it can be seen that, while for $\beta$-sources enhancements of up to $10 \%$ are attainable, for $\alpha$-sources only enhancements of $\sim 0.1-1 \%$ are possible.

Fig. 6 visualizes a classical spherically encapsulated RHU and the same RHU but promoting an internal electrostatic field in its interior or kernel.

\section{Summary results and conclusions}

In this paper the use of a self-induced electrostatic field has been proposed as a novel concept for power enhancement in radioisotope heat sources. Here the build-up of positive charges in a central kernel is used to generate a repulsive electrostatic field which will endow with additional kinetic energy the positively charged fragments arising from radioactive decay in the outer region. The main conclusions to be derived from this preliminary study are as follows:

(a) Self-electrostatic radioisotopic enhancement can yield an extra output power of up to $10 \%$ if $\beta$-decay sources are used.

(b) In contrast, it was deduced that self-electrostatic radioisotopic power enhancements are negligible if $\alpha$-decay sources are used, because of the reduced accumulation of positive

charge possible in the kernel due to the less energetic electrons in comparison with the $\beta$-decay source case.

(c) Self-electrostatic radioisotopic heat sources can be encapsulated and used to provide heat to keep structures, systems, and instruments warm enough to operate effectively, or used to produce electricity via thermal converters, e.g. through the Seebeck effect, but without needing to modify the overall structural design of the device, thus avoiding expensive adaptations.

(d) With the use of self-electrostatic radioisotopic heat sources, the attainable enhancement of the power output per unit volume can be used to reduce the amount of radioisotopic material used, and/or to increase the thermal efficiency in RTGs. Radioisotope mass reduction will be particularly significant in space exploration applications where payload reduction is a priority, and also because of the cost of certain radioisotopes due to their expensive production methods.

(e) The preliminary results in this paper are encouraging and motivate further research into this concept.

\section{Acknowledgements}

The first author is indebted to Engineer M. Scott for many helpful, encouraging, and stimulating discussions which helped him understand the problem. This research was supported by the National Science Foundation under Grant No-NCC-1701.

\section{Nomenclature}

$a$

$b$

e

$\mathbf{E}$

$\mathbf{G}_{e}$

$l_{e}$

$N_{0}$

$q$

$Q_{t}$

$r$

$W_{e}$

$\dot{W}_{e}$ radius of Region I

outer radius of Region II

electron energy (in electron-volts)

electrical field

electrostatic power gain

mean path length of the most energetic electron concentration of radioactive atoms per unit volume electron charge

total positive charge inside the kernel (Region I)

radial distance

electrostatic energy

electrostatic power 


\section{$W_{p} \quad$ decay energy \\ $\dot{W}_{p} \quad$ decay power \\ $\dot{W}_{t} \quad$ total power \\ $x \quad b / a$ \\ $z \quad$ number of electrical charges per disintegration}

\section{Greek symbols}

$\varepsilon_{0} \quad$ electrical permittivity in vacuum

$\lambda$ decay constant

$\rho \quad$ material density

$\rho_{+} \quad$ volumetric concentration of positive charges

$\Phi \quad$ electrical potential

\section{Subscripts}

$\begin{array}{ll}e & \text { electrostatic } \\ p & \text { decay } \\ m & \text { maximum } \\ t & \text { total }\end{array}$

$t \quad$ total

\section{References}

Anderson, D.J., Wong, W.A., Tuttle, K.L., 2005. An Overview and Status of NASA's Radioisotope Power Conversion Technology NRA. U.S. National Aeronautics and Space Administration, NASA/TM-2005-213981.

Arias, F.J., 2011. Advanced subcritical assistance radioisotope thermoelectric generator: an imperative solution for the future of NASA exploration. J. Brit. Inter. Soc. 64, 314-318.
Bejan, A., Sciubba, E., 1992. The optimal spacing for parallel plates cooled by forced convection. Int. J. Heat Mass Transf. 35, 3259-3264.

Corliss, W.R., Harvey, D.G., 1964. Radioisotopic Power Generation. Prentice-Hall, Englewood Cliffs, N.J.

Hajmohammadi, M.R., Pouzesh, A., Poozesh, S., 2012. Controlling the heat flux distribution by changing the thickness of heated wall. J. Basic Appl. Sci. Res. 2, $7270-7275$.

Hajmohammadi, M.R., Moulod, M., Joneydi Shariatzadeh, O.J., Nourazar, S.S., 2013. New methods to cope with temperature elevations in heated segments of flat plates cooled by boundary layer flow. J. Therm. Sci. (00) 159-159.

Hajmohammadi, M.R., Nourazar, S.S., Campo, A., Poozesh, S., 2013. Optimal discrete distribution of heat flux elements for in-tube laminar forced convection. Int. J. Heat Fluid Flow 40, 89-96.

Hajmohammadi, M.R., Poozesh, S., Rahmani, M., Campo, A., 2013. Heat transfer improvement due to the imposition of non-uniform wall heating for in-tube laminar forced convection. Appl. Therm. Eng. 61, 268-277.

Hajmohammadi, M.R., Maleki, H., Lorenzini, G., Nourazar, S.S., 2015. Effects of Cu and Ag nano-particles on flow and heat transfer from permeable surfaces. Adv. Powder Technol. 26, 193-199.

Katz, L., Penfold, A.S., 1952. Range-energy relations for electrons and the determination of beta-ray end-point energies by absorption. Rev. Mod. Phys. 24, 28-44.

Lazarenko, Y.V., Gusen, V.V., Pustovalov, A.A., 1988. Basic parameters of a radionuclide thermoelectric generator. Sov. At. Energy 64, 131-136.

Plonus, M.A., 1978. Applied Electromagnetics. McGraw-Hill, New York, N.Y.

Prelas, M.A., Weaver, C.L., Watermann, M.L., Lukosi, E.D., Schott, R.J., Wisniewski, D.A., 2014. A review of nuclear batteries. Prog. Nucl. Energy 75 117-148.

Radioisotope Power Systems Committee, National Research Council, 2009. Radioisotope Power Systems: an Imperative for Maintaining U.S. Leadership in Space Exploration. The National Academies Press, Washington, D.C.

Trenn, T.J., 1980. The phenomenon of aggregate recoil: the premature acceptance of an essentially incorrect theory. Ann. Sci. 37, 81-100.

Wang, S., He, C., 2014. Design and analysis of nuclear battery driven by the external neutron source. Ann. Nucl. Energy 72, 455-460. 